\title{
Using a mobile eye-tracking technology to explore pedestrians' gaze distribution on street space
}

\author{
Sergey Rudenko ${ }^{1}$, Nina Danilina ${ }^{*}$, and Borislav Hristov ${ }^{2}$ \\ ${ }^{1}$ Moscow State University of Civil Engineering, Yaroslavskoe shosse, 26, Moscow, 129337, Russia \\ ${ }^{2}$ University of Applied Sciences (HTW Berlin), Department of Engineering Sciences, Germany
}

\begin{abstract}
The paper deals with the question of smart sustainable city development on the example of public city street space study using a modern mobile eye-tracking technology. The article presents a methodology to obtain data on the distribution of human gaze on dynamic and static objects located on urban streets as well as computer technologies that are used for the acquisition and processing of data. With the help of these technologies, it is possible to study people's behavior in dynamic environments and to obtain results describing the nature of the pedestrians' perception of their surrounding space. The results of a survey on urban streets in Berlin using a mobile eye tracking system are shown in this paper. Based on the results of the study, a list of objects that catch people's attention and a numerical description of the gaze distribution are presented.
\end{abstract}

\section{Introduction}

Nowadays digital technologies can be used to answer urgent urban problems. This approach is the heart of the concepts of smart sustainable city, aimed at meeting the population needs in a comfortable and safe urban environment $[1,2,3]$. City streets in the 21 st century are becoming the centre of human activity, offering a variety of infrastructure and services $[4,5,6]$. One of the most interesting technologies that can be used to study the behavior of the population is eye-tracking. The technology of tracking a person's gaze allows us to obtain data on the distribution of human attention depending on the environment conditions $[7,8,9]$. There are existing studies of the drivers' gaze behavior while driving on highways on which many design guides and regulative norms were developed $[10,11,12,13]$. By this analogy, the study on pedestrian behavior also has a potential practical novelty and is interesting for research.

In international practice, studies on the perception of urban environment using eyetracking technology have already been conducted, such as the perception of surrounding buildings to determine placemaking aspects [14], the study of cyclists' gaze behavior in urban environment [15], the perception of public space boundaries by walking pedestrians [16].

\footnotetext{
* Corresponding author: grado@mgsu.ru
} 
The purpose of the study was to determine the features of the visual perception on city street public space infrastructure elements. In the current high-density buildings, saturated with various objects, the visual attention of pedestrians scatters on objects located along the route. The research question is which objects attract the attention of pedestrians. Thus, the object of the study is the urban space with placed infrastructure objects, which are visually perceived by persons in everyday life and has a direct impact on their psycho-emotional state and behavior.

The tasks of the research include the development of a methodology for studying the visual perception of city street space by pedestrians, the study of the features and patterns of visual perception of various infrastructure objects located on city streets, and the analysis of the obtained results.

The scientific novelty of the research lies in the fact that the urban space was not studied at laboratory conditions where all subjects are shown a static stimulus in the form of an image, but the subjects are directly in the surrounding urban space and the stimulus is a dynamic scene.

\section{Methodology}

The research methodology included a thorough selection of stimuli to achieve the goal and objectives, taking into account the requirements for the scientific reliability of the results.

The research methodology presents the following sequence (figure 1).

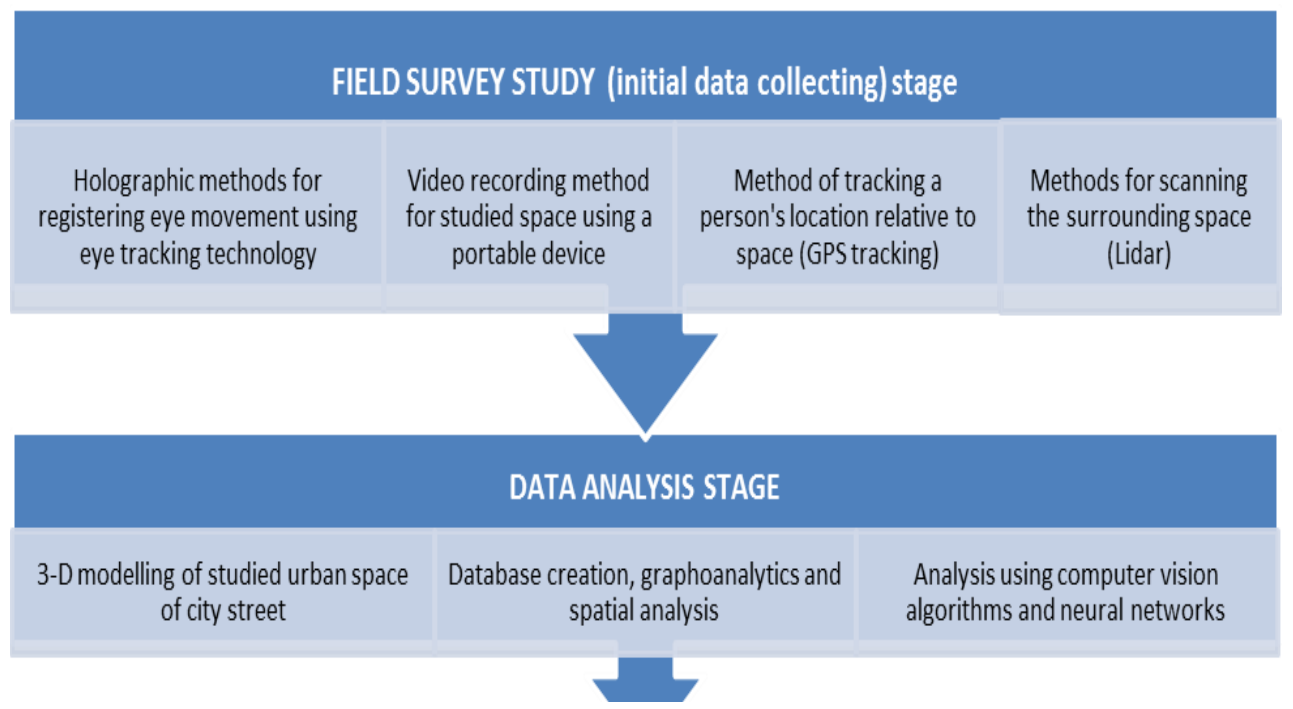

Fig. 1. Methodology of city street public space research using a mobile eye-tracking technology.

The initial data was collected in the field survey using the Tobii Pro Glasses 2 eyetracker. This invention relates to non-invasive oculography using infrared illumination of the cornea. Simultaneously with the registration of eye movement metrics, audio and video recordings of the surrounding urban space were performed. The device has the technical characteristics given in Table 1 .

The city street Wilhelminenhofstraße, located in the Oberschöneweide district of Berlin, Germany, was chosen as object of the study (Fig. 2). Wilhelminenhofstraße is a local street with a fairly high number of pedestrians and passenger traffic due to its location near the University of Applied Sciences (HTW Berlin), which has about 10,000 students on this particular campus. A weekday at lunchtime was chosen as the study period. During the 
study, the number of pedestrians and the occupancy of public transport stops changed periodically.

Table 1. Technical specifications of "Tobii Pro Glasses 2"

\begin{tabular}{|c|c|}
\hline Data & Characteristics \\
\hline Sample Rate & 50 or $100 \mathrm{~Hz}$ \\
\hline Calibration procedure & One point \\
\hline Calibration validation check & Available \\
\hline Parallax compensation tool & Automatic \\
\hline Slippage compensation & Available, 3D eye tracking model \\
\hline Eye tracking technique & Corneal reflection, binocular, dark pupil \\
\hline Measurement of the pupil & Available. Absolute measure \\
\hline Number of eye tracking sensors & Gyroscope and accelerometer \\
\hline Sensors & Available, 1920 x 1080 pixels 25 frames per \\
second, H. 264
\end{tabular}

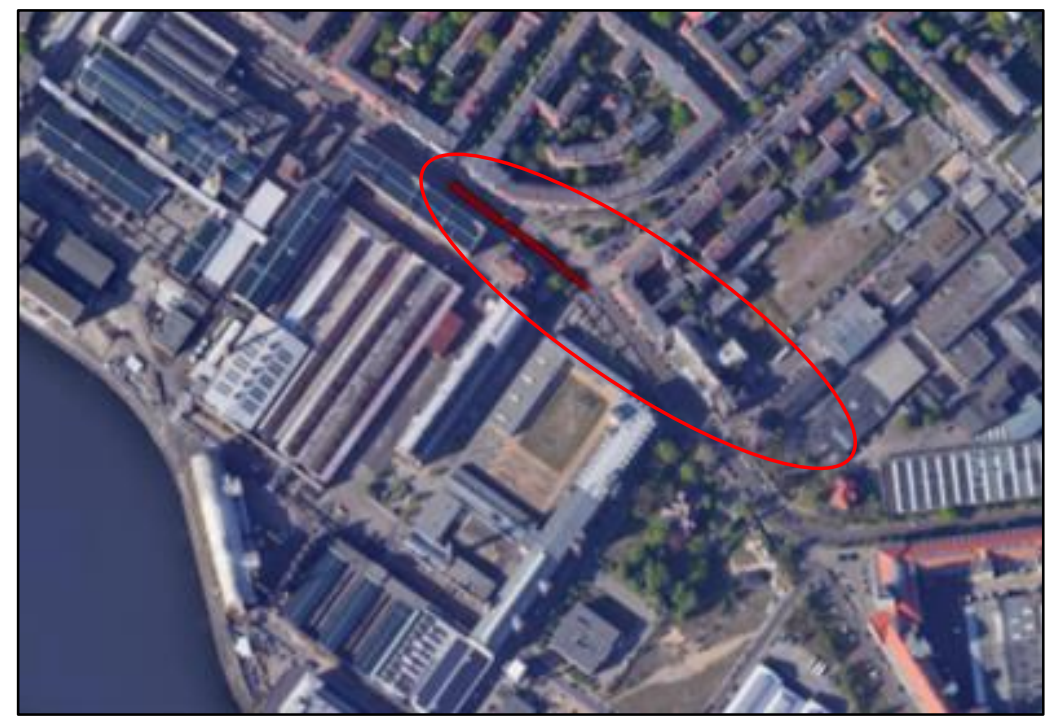

Fig. 2. Research section of the city street Wilhelminenhofstraße in Berlin, Germany.

Eight subjects aged 19-30 years - students and employees of the MGSU took part in the process of initial data collecting. All subjects had no idea about the goals of the experiment. Their task was to go along the street and return back as they would do in normal life walking along the same section of the street. During the passage, their eye movements were recorded by the eye tracking glasses in the metric data recording module. After that, the recorded data was imported into the program "Tobii Pro Lab" and processed.

The hypothesis of the study was that different subjects in the experiment fixate on the same infrastructure objects. The study of the color, geometry, and location of the object that 
attracts the pedestrian's gaze will allow us to identify general trends in people's perception of the city street space, which can form the basis for developing recommendations for their arrangement. Using the tools of the Tobii Pro Lab software, the eye fixation metrics were projected onto the recorded video (Fig. 3 a) and the data was visualized as a "heat map", as well as a "sequence and duration map" of fixations including their scan paths (Fig. 3 b,c).

a)

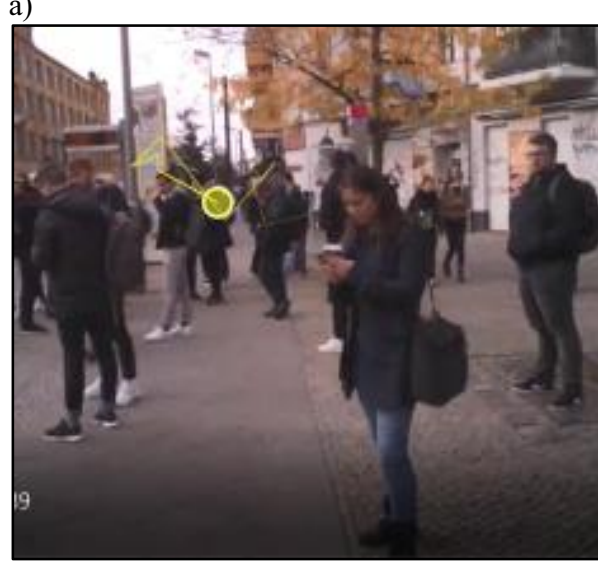

c)

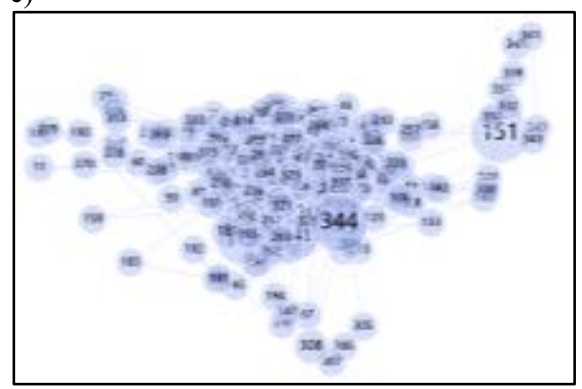

b)

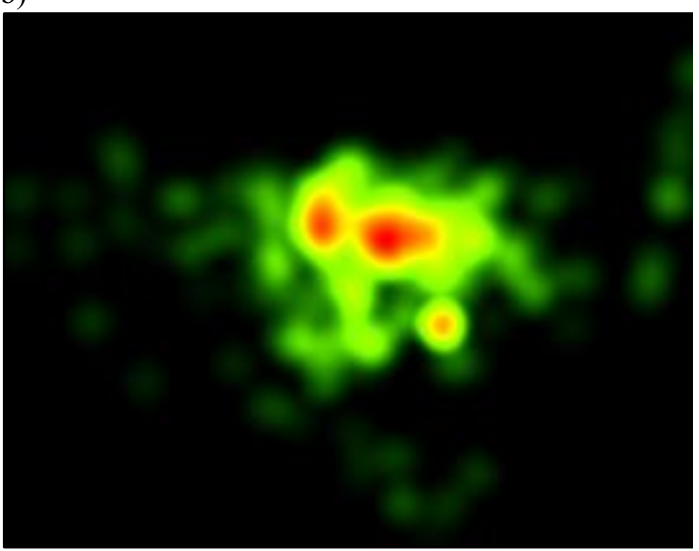

Fig. 3. Tools of the software "Tobii Pro Lab".

a) fragment of projecting motion metrics

b) Example of a "heat map"

c) a map of the sequence and duration of fixations with their scan paths

At the stage of data evaluation, we made an analysis of the video sequence using "Adobe Premiere program".

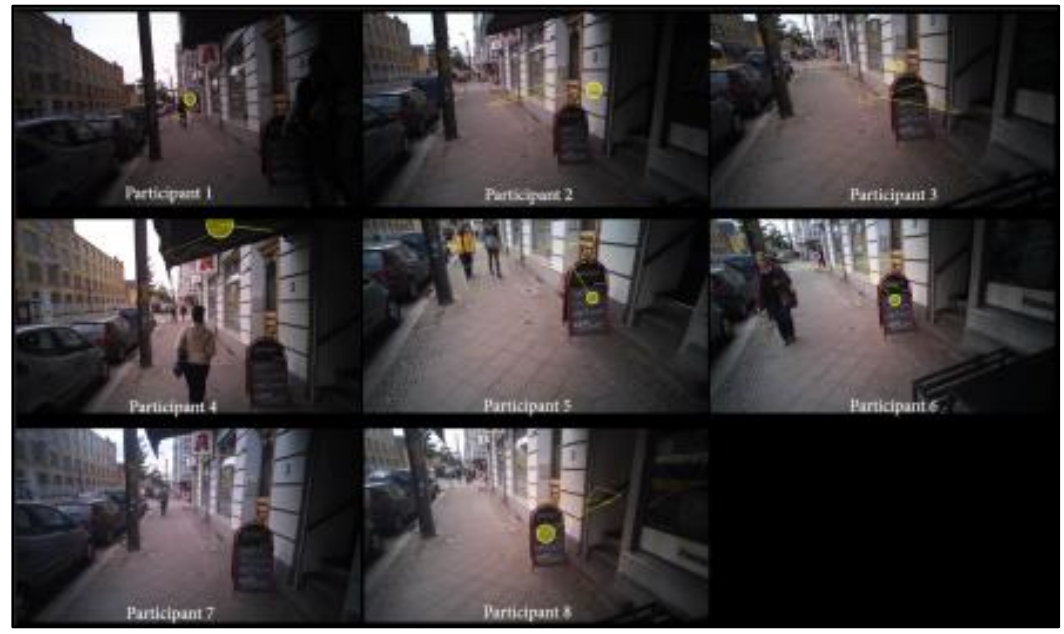

Fig. 4. Example of fixing the subjects' view on an object. 
The recorded videos with the projection of each subject's metrics were imported and placed in a single window. Further, individual fragments of the video tracks were edited to achieve synchronicity of the subject's location on the video at the same time, because the movement speed of the subjects was different. After achieving synchronicity of movement in the video, it was found that some subjects fixated on the same object in the surrounding space (Fig. 4).

For collected data processing Tobii Pro Lab equipment was used to export the metric data of each subject to a table with a ".tsv" file extension. After that, additional columns were added to the existing table with the description of the qualitative and quantitative characteristics of the objects fixed by the view of each subject.

At the next stage, the video sequence of each subject was decomposed into frames, which were then saved in separate files with a ".jpg" file extension. In the process of saving the frame, information from the metric data table was projected onto it and recorded using code written in the Python programming language using the Opencv and Numpy libraries (Fig. 5).

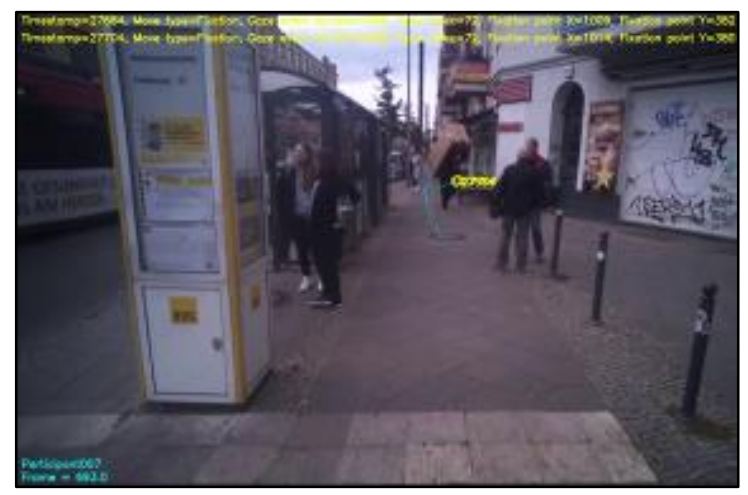

Fig. 5. Example of a saved video frame with recorded metric data.

The next stage was to place the point of fixation of the view in three-dimensional space in order to obtain coordinates for subsequent spatial analysis. The implementation of this task was performed in the "Rhinoceros" three-dimensional graphics editor in the following sequence:

1. Positioning of GPS points with a timestamp as the attribute value for identification;

2. Placement of fixation points with a timestamp as the attribute value for identification;

3. Export the geometry of the fixation points and GPS points to a file that describes the coordinates of these points.

A fragment of the arranged fixation points in Rhinoceros is shown in figure 6. 


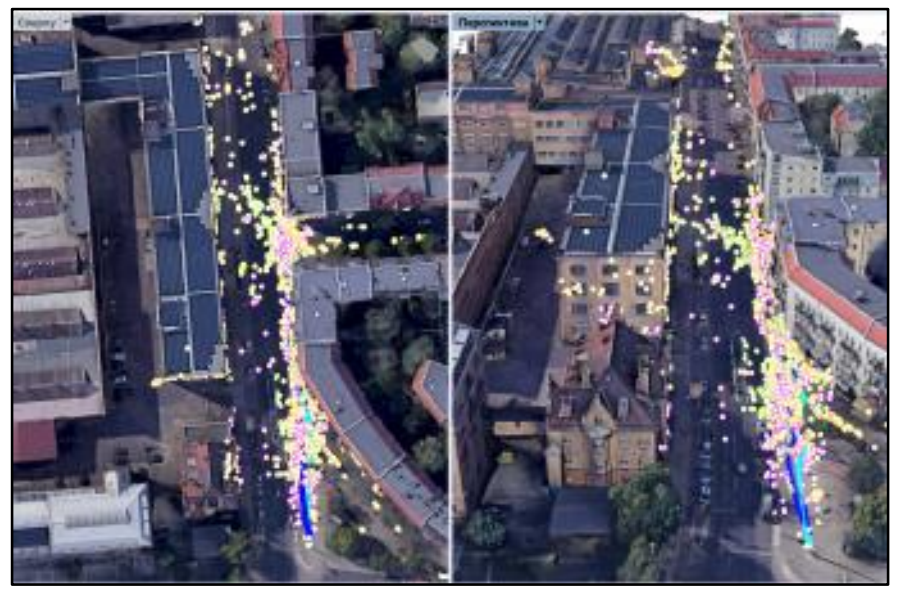

Fig. 6. A fragment of the fixation points using "Rhinoceros" program.

At the final stage processing data was loaded in the PostgreSQL database as:

- Metric data of each subject's eye fixations;

- Description of the qualitative and quantitative characteristics of the fixated objects;

- Fixed points and GPS points in the form of 3D geometry in WKT format;

- Frames with a projection of metric data.

As a result, previously disparate data was organized and linked in a single relational model that has ample manipulation capabilities and high operation speed for this amount of data. After loading the data into the database, various queries were performed in the SQL language in order to obtain statistical and spatial analysis of the data, for example, spatial analysis of the fixation points and the initial position of a person in space for a differentiated estimation of the distance to the objects of fixation.

The final stage was the interpretation of the results. The resulting diagrams were compiled using Python and the Matplotlib library, which has a powerful data visualization tools. The data interpretation process has the following sequence:

1. SQL query to the database using the psycopg2 library;

2. Processing query results in Python;

3. Output of various diagrams using the Matplotlib library.

The "heat maps" of fixation points in space were obtained by means of QGIS and the HeatMap plugin, which calculates the density of fixation points in a specific location based on the kernel density estimation KDE method, with a radius of 5 meters and the Epanechnikov nuclear function as parameter values.

\section{Results}

The results of the study included consideration of two aspects:

- The patterns of a person's gaze distribution when walking along a city street;

- Statistics of pedestrian perception of various infrastructure objects located on city streets.

Let's take a closer look at each of the points.

Figure 7 shows the fixation distribution with a high fixation density in the central part of the projection and a decreasing density to the edges of the projection, measured in the Display Area Coordinate System (DACS) of the projection points. It should be noted that the scatterplot does not take into account the rotation of the subject's head. Based on this feature, it can be concluded that the subjects' zone of interest is in the central part of the 
projection in order to comfortably perceive the object, but obviously, a small part of the fixations were also perceived by the peripheral vision at the edges of the projection.

Based on this feature, it can be concluded that subjects placed the area of interest in the central part of the projection in order to comfortably perceive the object, but as we see a small portion of fixations were also perceived at the edges of the projection, such objects were perceived by the peripheral vision.

The diagram of the number of fixations in figure 8 shows that most of the fixations last between $80 \mathrm{~ms}$ and $500 \mathrm{~ms}$ and fixations with a duration of $500 \mathrm{~ms}$ to $2 \mathrm{~s}$ are extremely rare. Consequently, pedestrian subjects who are in motion are more likely to fixate their gaze on an object for up to $500 \mathrm{~ms}$, and in rare cases keep their attention on the object of interest for up to $2 \mathrm{~s}$. The data on the dependence of the amount and duration show that most of the information is perceived by the subjects in fairly short periods of time.

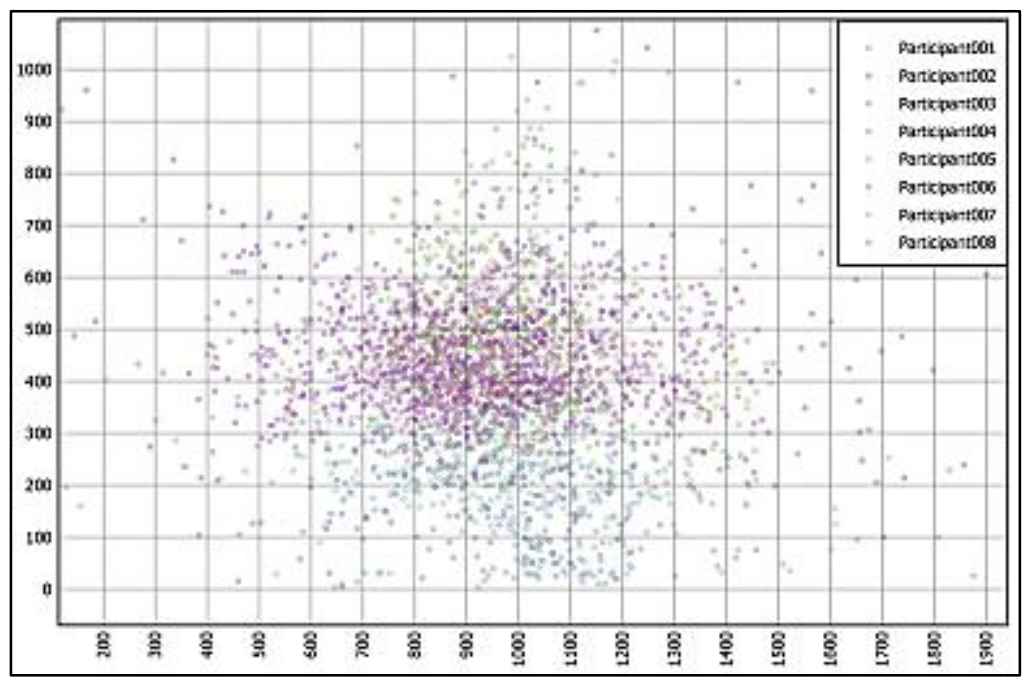

Fig. 7. Scatterplot of the fixation distribution on the projection.

The distribution in figure 9 shows the total fixation durations for each dynamic or static objects type in the street space. As a result of analyzing the obtained data, a list of the objects attracting subjects' was established. It is noted that most of the fixations fall on dynamic moving people. As for the static infrastructure objects located on the street space, the subjects mostly fix their attention on the sidewalk rather than on the roadway. Subjects' focus lied on determining whether there were obstacles in their path. After being convinced that there is no obstacle on the way, subjects fix their eyes on the facades of buildings.

Thus, if we rank the objects of interest in descending order, the sequence will look like this:

1. People;

2. Sidewalk pavement;

3. Buildings and structures;

4. Infrastructure elements;

5. Roadway and transport. 


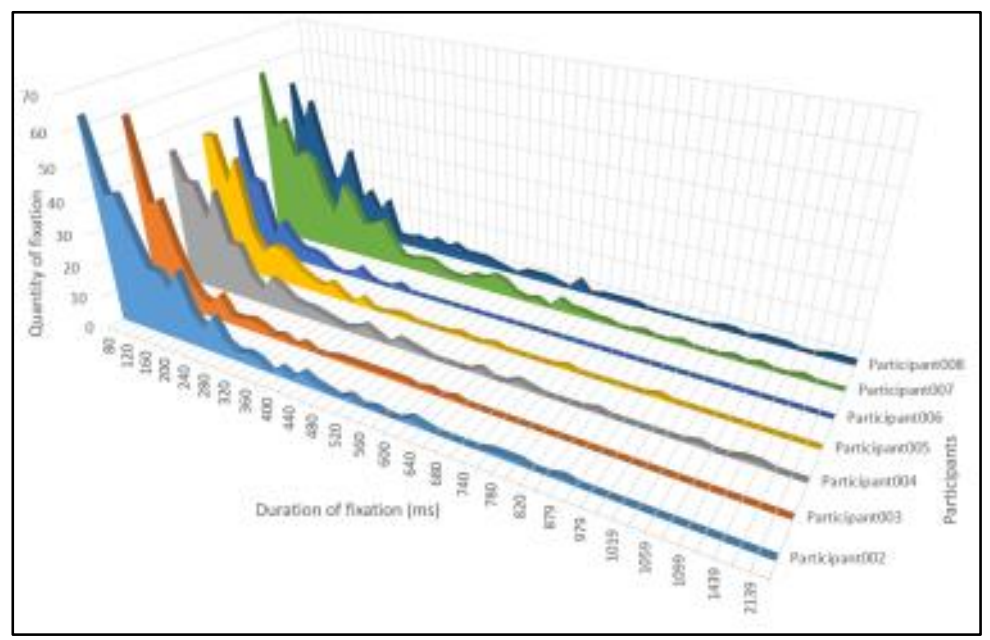

Fig. 8. Distribution of the number of fixations by their duration.

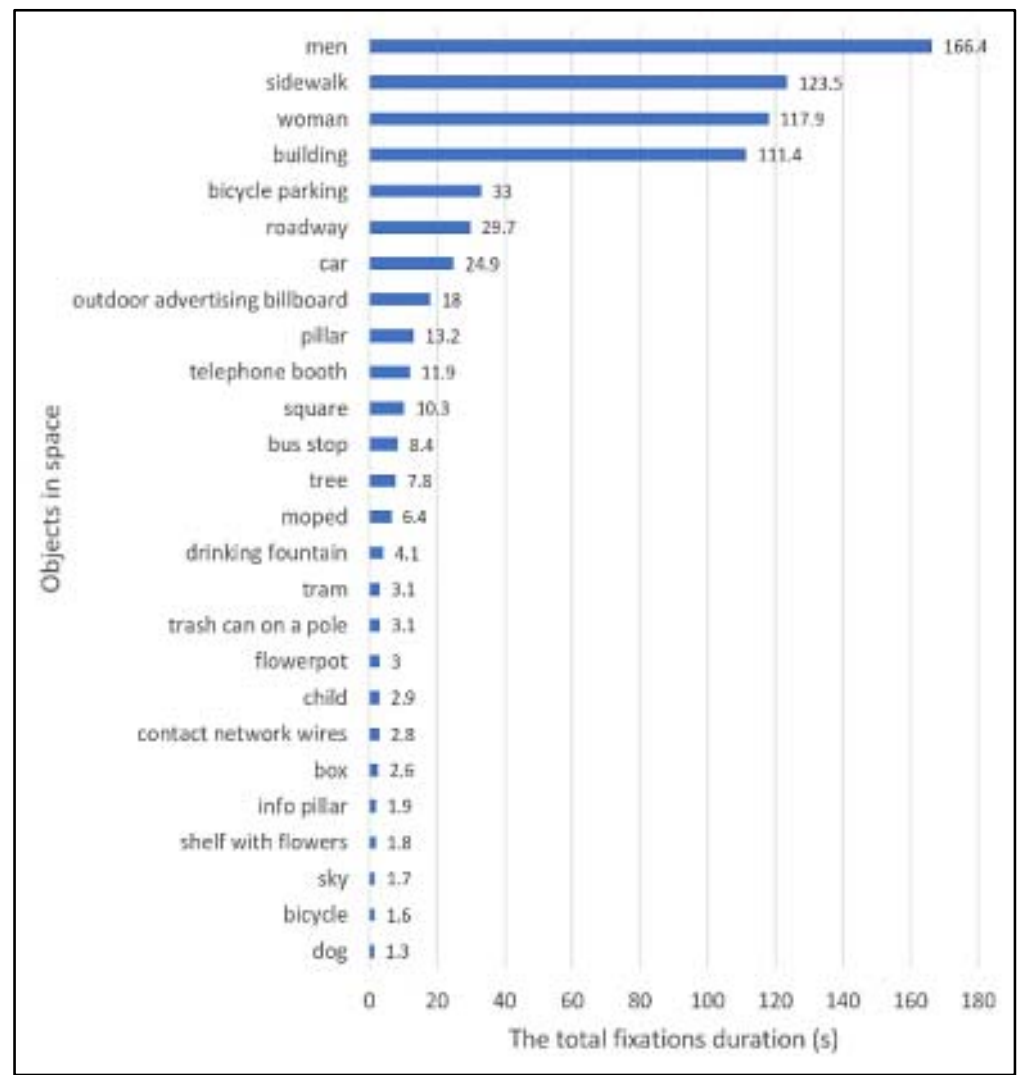

Fig. 9. Distribution of fixation durations for different objects in the street space. and 


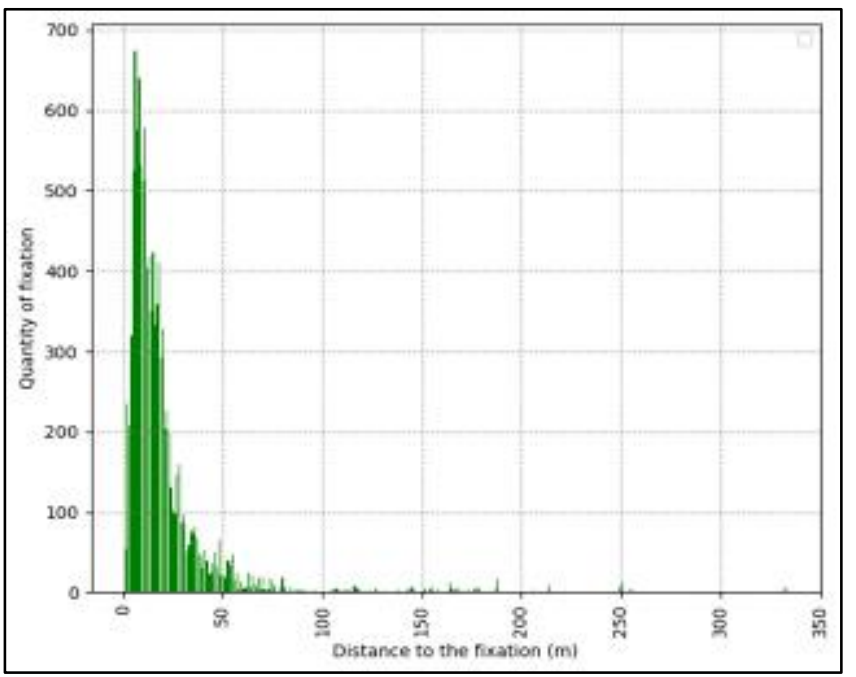

Fig. 10. distribution of the number of fixations depending on the distance between test person and fixated object.

While walking along the street, the subjects fixated on objects located at different distances from the subjects' location, which range is between 1 and 333 meters. The diagram in figure 10 shows that the majority of fixations falls at distances of 1-55 meters, whereas the peak quantity lies in the range of 5-10 meters. A decrease is observed between 55 and 200 meters and fixations laid at distances $200-333$ meters are very rare. The revealed features show that the study subjects in the vast majority receive information about the object of interest from a distance in the range of 5-10 meters. This dependence should be taken into account, for example, when placing information signs of navigation systems for the orientation of pedestrians in the city.

The "heat map" in the figure 11 shows the density of fixation points on the plan of the street test section, indicating the zones where the objects that cause the greatest interest and focus of attention of the subjects are located. The analysis of such zones shows that they contain objects that are a potential obstacle to the subjects' journey, such as outdoor billboards, the construction of public transport stops, lighting poles and outdoor furniture of outdoor cafes. In rare cases, areas with a high density of fixation contain objects that do not interfere with the movement of subjects, such as advertising structures on building facades and stained glass windows.

It is worth noting that on the roadway of the street, which is the main source of danger, the vehicles were rarely fixed, and this fact indicates the comfort of the available width of the sidewalk. 


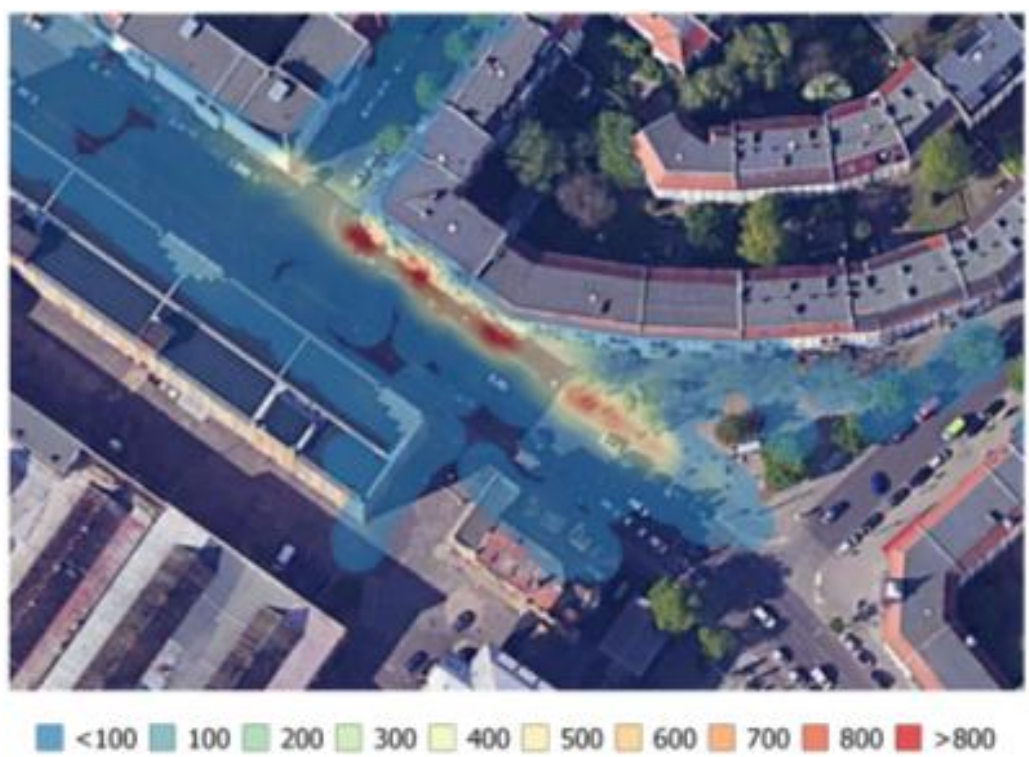

Fig. 11. Heat map of the density of fixations on the plan of the street test section. The values reflect the amount of fixations in a given location.

The obtained data is a basis for the development of practical recommendations for street design. Through the distribution of the pedestrians' gaze it is possible to choose the right placement of different objects on sidewalks and the materials and façade characteristics can be made. A very important outcome of this research is the need of a linear zoning of the sidewalk space on city streets to place various objects for different purposes, in order to ensure a comfortable psycho-emotional pedestrians' perception of urban space.

\section{Conclusion}

Urban environment research using a mobile eye-tracking technology presents a wide range of topics for further research. The results obtained in this study certainly require further elaboration and accumulation of empirical data under different experimental conditions in subsequent studies in order to increase sampling and statistical representativeness. Also, the prospect is that introduction of an interdisciplinary approach and additional involvement of methods of social, behavioral, environmental research will provide successful results in the field of people's perception of the urban environment. The presented results are of practical value for the development of recommendations for improvement of city street spaces and organization of comfortable and safe conditions for pedestrian traffic. This approach should be the main one for improving the quality of the urban environment as basis for the development of smart sustainable cities.

\section{References}

1. N. Danilina, A. Majorzadehzahiri IOP Conf. S.: Materials Science and Engineering. 869 (2). 022027 (2020)

2. T. Yigitcanlar, Md. Kamruzzaman Land Use Policy. 73. (2018) 
3. Chuanjun Zheng, Jingfeng Yuan, Lei Zhu, Yajing Zhang, Qiuhu Shao Jour. of Cleaner Production. 258. 120689 (2020)

4. K. Carlotta von Schönfeld, L. Bertolini Cities. 68, 48-55 (2017)

5. N. Danilina, S. Privezentseva IOP Conf. S.: Mat. Sc. and Eng. 775, Iss. 1, 012008 (2020)

6. Wesley E. Marshall, Daniel P. Piatkowski, Norman W. Garrick Jour. of Transport \& Health. 1, Issue 4, 326-340 (2014)

7. Jolene A. Cox, Anne M. Aimola Davies Neuropsychologia, 146, 107547 (2020)

8. H.M. Fehd, A. E. Seiffert Cognition, 108, Iss.1, 201-209 (2008)

9. Min Zhang, Ruo-Xi Zhang, Tay-Sheng Jeng, Zi-Yuan Jour. of Visual Commun. and Image Repres., 64, 102639 (2019)

10. B. Hristov XXII Int. Sc. Conf. FORM-2019, 97 (2019)

11. B. Hristov Dissertation at the Technical University of Dresden, Faculty of Transportation Sciences (2010)

12. M. N. Russi-Vigoya, P. Patterson Proc. Manufac., 3, 5028-5035 (2015)

13. D. Vetturi, M. Tiboni, G. Maternini, M. Bonera Transp. Res. Proc., 45, 587-595 (2020)

14. Hollander, Justin \& Purdy, Alexandra \& Wiley, Andrew \& Foster, Veronica \& Jacob, Robert \& Taylor, Holly \& Brunyé, Tad Journal of Urbanism: International Research on Placemaking and Urban Sustainability. 12. 1-16 (2018)

15. Mantuano, Alessandra \& Bernardi, Silvia \& Rupi, Federico Case Studies on Transport Policy. 10. (2016).

16. J. Simpson, K. Thwaites, M. Freeth Sustainability, 11, 4251 (2019). 